\title{
Analysis of MERCATOR data - Part I: variable B stars
}

\author{
P. De Cat ${ }^{1,2}$, M. Briquet ${ }^{2,6}$, C. Aerts ${ }^{2,3}$, K. Goossens ${ }^{2}$, S. Saesen ${ }^{2}$, J. Cuypers ${ }^{1}$, K. \\ Yakut $^{2}$, R. Scuflaire ${ }^{4}$, M.-A. Dupret ${ }^{5}$ and many observers \\ ${ }^{1}$ Royal Observatory of Belgium, B-1180 Brussel, Belgium \\ 2 Instituut voor Sterrenkunde, Katholieke Universiteit Leuven, B-3001 Leuven, Belgium \\ ${ }^{3}$ Department of Astrophysics, Radboud University Nijmegen, 6500 GL Nijmegen, the Netherlands \\ 4 Institut d'Astrophysique et de Géophysique, Université de Liège, B-4000 Liège, Belgium \\ ${ }^{5}$ Observatoire de Paris, LESIA, 92195 Meudon, France
}

\begin{abstract}
We re-classified 31 variable $B$ stars which were observed more than 50 times in the Geneva photometric system with the P7 photometer attached to the MERCATOR telescope (La Palma) during its first 3 years of scientific observations. HD 89688 is a possible $\beta$ Cephei/slowly pulsating B star hybrid and the main mode of the COROT target HD 180642 shows non-linear effects. The Maia candidates are re-classified as either ellipsoidal variables or spotted stars. Although the mode identification is still ongoing, all the well-identified modes so far have $\ell \leq 2$.
\end{abstract}

\section{Introduction}

The Mercator telescope is a 1.2-m telescope located on the Roque de los Muchachos observatory on La Palma (Spain). Since the start of scientific observations in spring 2001, this telescope has been intensively used to observe variable $B, A$ and $F$ main sequence stars with the P7 photometer, providing quasi-simultaneous observations in the 7 passbands of the Geneva photometric system. The first results obtained after 18 months of observations were already presented by De Cat et al. (2004) and De Ridder et al. (2004). We now present results after 3 years of collecting data. In Part I (this paper), the analysis of the 9023 datapoints of variable B stars is discussed while Paper II (Cuypers et al., these proceedings) focuses on the analysis of the 5149 datapoints of variable $A$ and $F$ stars.

We here restrict ourselves to the 31 variable $B$ stars which were not included in multi-site campaigns and which were observed at more than 50 epochs. Based on the photometric observations gathered with the satellite mission HIPPARCOS, these objects were previously classified as candidate $\beta$ Cephei stars ( $\beta$ Ceps; main-sequence B $0-3$ stars pulsating in low order, low degree $p / g$-modes with periods of 3-8 h), slowly pulsating B stars (SPBs; mainsequence B 3-B 9 stars pulsating in high order, low degree $g$-modes with periods of $0.5-3 \mathrm{~d}$ ) and Maia stars (Maias; variable main-sequence stars situated between the SPBs and the $\delta$ Scuti stars). They are respectively given with squares, triangles and stars in Fig. 1.

\footnotetext{
${ }^{6}$ Postdoctoral Fellow of the Fund for Scientific Research, Flanders
} 


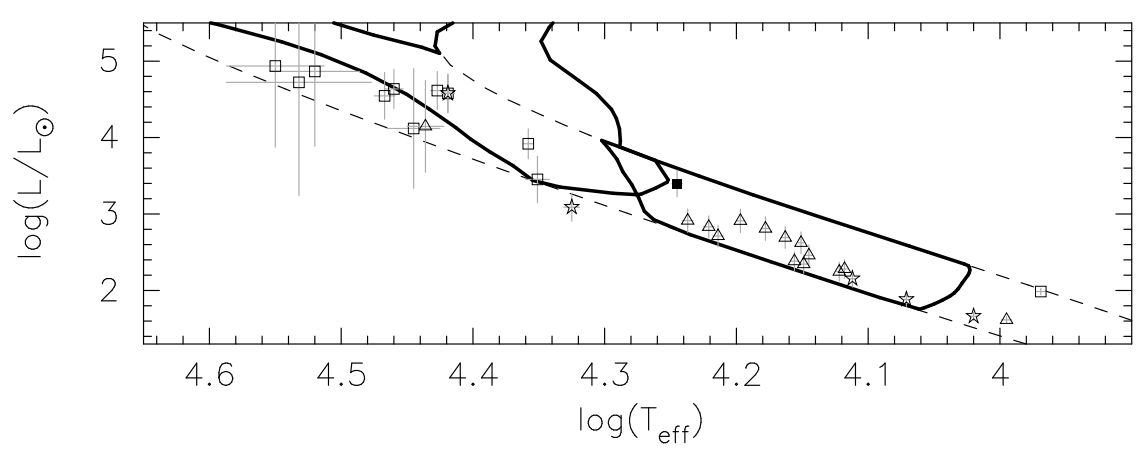

Figure 1: Position in the H-R diagram of the 31 variable $B$ stars discussed in this paper. The candidate $\beta$ Cephei stars, slowly pulsating $B$ stars and Maia stars are given with squares, triangles and stars respectively. HD 89688 is given with a full symbol. The ZAMS and TAMS are given with dashed lines, and the theoretical instability strips for $\beta$ Cephei and slowly pulsating B stars, as given by Pamyatnykh (1999), with full lines.

\section{Frequency analysis}

The time series in the Geneva passbands and colours were both subjected to a detailed frequency analysis with the PDM (Stellingwerf 1978) and Lomb-Scargle (Scargle 1982) methods. Since our ground-based data-sets suffer from strong aliasing, the space-based photometric observations of the HIPPARCOS satellite proved to be very useful to extract the physical frequencies in some cases. Our results enable us to re-classify the stars into the following categories by using the same criteria as De Cat et al. (2004):

- SPBs: 11 multiperiodic (HD 1976, 3379, 21071, 25558, 28114, 28475, 179588, 182255, 191295, 206540, 222555), and 2 monoperiodic (HD 138003, 208057)

- $\beta$ Ceps: 6 multiperiodic (HD 13745, 13831, 14053, 21803, 180642, 203664)

- Hybrid star: HD 89688

- Spotted stars: HD 46005, 154689, 169820

- Ellipsoidal variables: HD 24094, 112396, 149881, 208727

- Be star: HD 180968

- Constant stars: HD 19374, 214680, 217782

For all the periodograms and phase diagrams, we refer to De Cat et al. (in preparation). For HD 89688, we now have marginal evidence for the SPB-like frequency $0.7965(6) \mathrm{d}^{-1}$ (or one of its aliases), while the HIPPARCOS photometry points towards $\beta$ Cep-like frequency 7.3902(5) $\mathrm{d}^{-1}$. Its position in the $\mathrm{H}-\mathrm{R}$ diagram is compatible with the classification as a hybrid star (full symbol in Fig. 1). For the multiperiodic COROT target HD 180642, the first mode is a high amplitude mode which shows non-linear effects. We detect up to the second harmonic of $\nu_{1}=5.486971(6) \mathrm{d}^{-1}$, making it only the second $\beta$ Cep star for which more than one harmonic is observed (Aerts et al., in preparation). Note that for all the Maias, i.e. HD 46005, 154689, 169820, 208727, the observed variations are explained by mechanisms other than pulsations.

\section{Mode identification}

For the mode identification, we applied the method of the photometric amplitudes (Dupret et al. 2003) in which the observed and theoretical amplitude ratios relative to the amplitude 


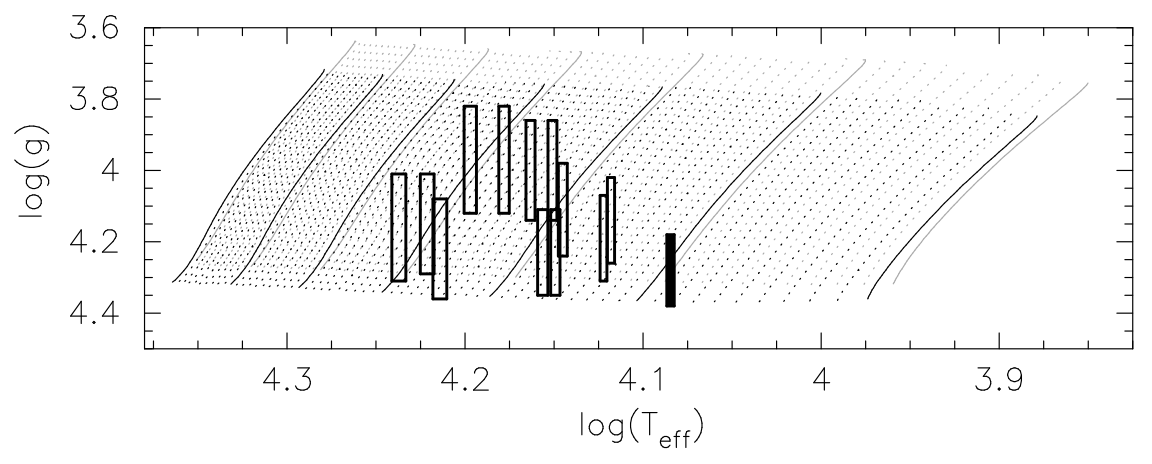

Figure 2: Presentation of the grids of equilibrium models used for the mode identification of the SPBs. The evolution tracks of grid 1 and 2 (see text) with masses between 2-8 $M_{\odot}$ in steps of $0.1 M_{\odot}$ are respectively given in light and dark grey. The boxes represent the observed error boxes of the 13 SPBs in our sample. The filled box corresponds to HD 179588.

in the $U$ filter are compared. For the SPBs, we confronted the results based on 2 grids of equilibrium models (Fig. 2). Grid 1 consists of models calculated with CLES-013 (written by R. Scuflaire) with an initial mass fraction of metals $Z_{0}=0.020$ and of hydrogen $X_{0}=$ 0.70 , mixing-length $\alpha_{\text {conv }}=1.80$, and the standard metal mixture of Grevesse \& Noels (1993). Grid 2 was obtained with CLES-018.2 with the 'new' solar values $Z_{0}=0.015$, $X_{0}=0.71, \alpha_{\text {conv }}=1.75$ and the standard metal mixture of Asplund et al. (2005). In both cases, we used the CEFF equation of state (Christensen-Dalsgaard \& Däppen 1992) and a Kurucz atmosphere with the junction point at optical depth $\tau=10$, and we assumed neither convective overshooting nor diffusion. One of the main changes between CLES-013 and CLES-018.2 is the use of the new value of the cross section of ${ }^{14} N(p, \gamma){ }^{15} O$ recently measured by Formicola et al. (2004). We calculated the non-adiabatic eigenfunctions and eigenfrequencies for $g$-modes with $\ell \leq 3$ with the code MAD (written by M.-A. Dupret). For each star, we selected the models within the observed error box of $\log \left(T_{\text {eff }}\right)$ and $\log g$ (boxes in Fig. 2), and selected the eigenfrequency which is the closest to the observed frequency to calculate the theoretical amplitude ratios. In Fig. 3, we give a representative example of our results, i.e. for the two main modes of HD 179588. Although there are significant differences in the position and/or the shape of the theoretical curves for the higher degree modes of grid 1 (left) and 2 (right), the identification of the modes remains the same, i.e. $\ell=1$ or 2 for the mode corresponding to $\nu_{1}=0.856543(15) \mathrm{d}^{-1}$ (top), and $\ell=1$ for the mode corresponding to $\nu_{2}=2.04263(5) \mathrm{d}^{-1}$ (bottom). In general, these differences coming from the use of 2 different grids increase for increasing values of the observed frequency. So far, all the well-identified SPB modes have $\ell=1$ or 2 . For the $\beta$ Ceps, the mode identification is still ongoing.

\section{Conclusions}

Our photometric survey allowed a significant contribution to the classification of variable $B$ stars. HD 89688 is a possible $\beta$ Cep/SPB hybrid star and the COROT target HD 180642 is a multiperiodic $\beta$ Cep star of which the main mode shows non-linear effects (Aerts et al., in preparation). Amongst the 31 targets with a sufficient amount of data, we identified 4 ellipsoidal variables and 4 spotted stars. Their classification should be checked by supplementary spectroscopic observations. The mode identification is still ongoing, but all well-identified 

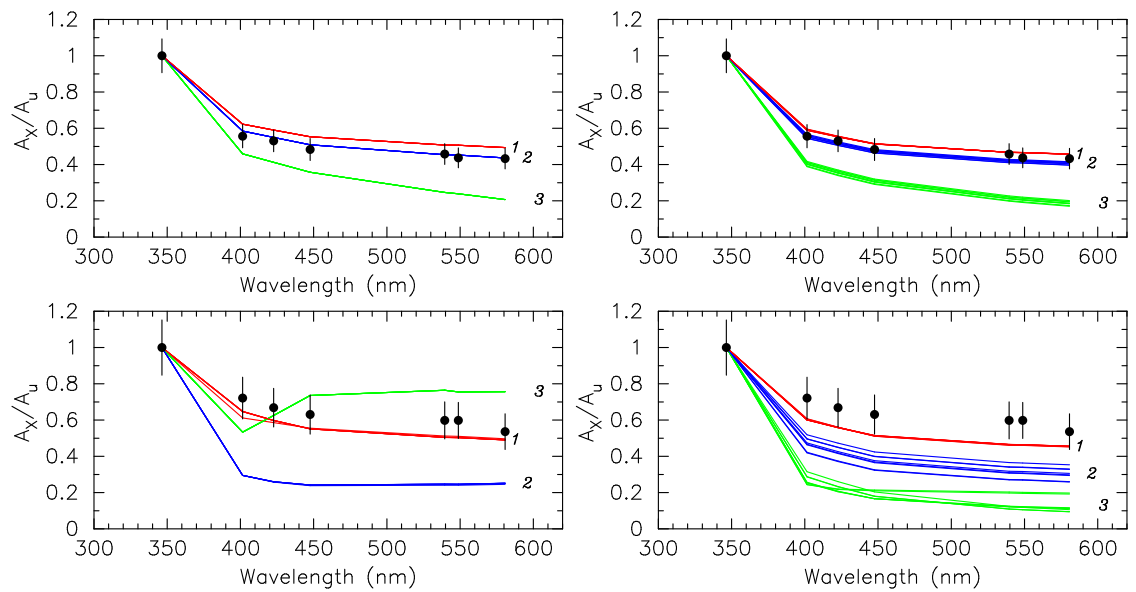

Figure 3: Photometric mode identification for $\nu_{1}=0.856543(15) \mathrm{d}^{-1}$ (top) and $\nu_{2}=2.04263(5) \mathrm{d}^{-1}$ (bottom) of HD 179588. For each theoretical model within the observed range of $\log \left(T_{\text {eff }}\right)$ and $\log g$, the theoretical amplitude ratios for modes with $\ell=1$ (full lines), 2 (dashed lines), and 3 (dotted lines) are represented with an individual line. The dots indicate the observed amplitude ratios and their standard error. The left and right panels show the results obtained with grid 1 and 2 respectively (see text).

modes have $\ell \leq 2$ so far.

The final results of our survey will be given by De Cat et al. (in preparation). The MERCATOR observations allow to take the first steps in asteroseismic modeling for two multiperiodic $\beta$ Cep stars, i.e. HD 203664 (Aerts et al. 2006) and HD 21803 (Saesen et al., in preparation). For $12 \mathrm{Lac}$ and $\mathrm{V} 2052 \mathrm{Oph}$, the MERCATOR telescope was included in multi-site campaigns. The data of these objects are being analysed by Handler et al. (submitted to MNRAS) and Handler et al. (2006) respectively.

Acknowledgments. This work is based on observations collected with the P7 photometer attached to the MERCATOR telescope (La Palma, Spain). We are very much indebted to all the observers from Leuven University. CA and JC acknowledge support from the Fund for Scientific Research (FWO) - Flanders (Belgium) through project G.0178.02.

\section{References}

Aerts, C., De Cat, P., De Ridder, J., et al. 2006, A\&A, in press (astroph/0511306)

Asplund M., Grevesse N., Sauval A.J. 2005, in "Cosmic Abundances as Records of Stellar Evolution and Nucleosynthesis", eds. Barnes III, T.G. \& Bash, F.N., ASP Conf. Ser. 336, 25

Christensen-Dalsgaard J., Däppen W. 1992, A\&A Rv 4, 267

De Cat, P., De Ridder, J., Uytterhoeven, K., et al. 2004, in "Variable Stars in the Local Group", eds. D.W. Kurtz, K.R. Pollard, ASP Conf. Ser. 310, 238

De Ridder, J., Cuypers, J., De Cat, P., et al. 2004, in "Variable Stars in the Local Group", eds. D.W. Kurtz, K.R. Pollard, ASP Conf. Ser. 310, 263

Dupret M.-A., De Ridder J., De Cat P., et al. 2003, A\&A 398, 677

Formicola A., Imbriani G., Costantini H., et al. 2004, Physics Letters B 591, 61

Grevesse N., Noels A. 1993, Physica Scripta 47, 133

Handler, G., et al. 2006, MNRAS, in press

Pamyatnykh, A.A. 1999, Acta Astr. 49, 119

Scargle, J.D. 1982, ApJ 263, 835

Stellingwerf, R.F. 1978, ApJ 224, 953 